\title{
Effects of Chemical Process on Dissipative Radiative MHD Flow through a Porous Medium over a Non-Isothermal Stretching Sheet with Heat Generation
}

\author{
M. Venkateswarlu ${ }^{1}$, G.V. Ramana Reddy ${ }^{2}$ and D.V.Lakshmi ${ }^{3}$ \\ ${ }^{I}$ Department of Mathematics, V.R.Siddhartha Engineering College, Kanur, Krishna (Dt,),A,.India,Pin-520007 \\ ${ }^{2}$ Department of Mathematics, K. L.University, Guntur (Dt),A.P,India,Pin -522502 \\ ${ }^{3}$ Department of Mathematics, Bapatla women Engineering College, Bapatla,Guntur (Dt),A.P,India,Pin-522101
}

\begin{abstract}
The steady two dimensional radioactive MHD boundary layer flow of an electrically viscous, incompressible, conducting fluid caused by a non-isothermal linearly stretching sheet placed at the bottom of fluid saturated porous medium in the presence of viscous dissipation, heat generation and chemical reaction is studied. The governing system of partial differential equations is converted to ordinary differential equations by using the similarity transformations and then solved by shooting method. The dimensionless temperature, velocity, and concentration are computed for different thermo physical parameters viz the magnetic parameter, permeability parameter, , wall temperature parameter radiation parameter, Prandtl number, heat generation parameter, Eckert number, Schmidt number and chemical reaction.
\end{abstract}

Keywords: Chemical reaction, Eckert number, Permeability parameter, Porous medium, Shooting method.

\section{INTRODUCTION}

The fluid flows with chemical reaction have attracted the attention of engineers and scientists in the present times. This kind of flows have key importance in many processes including drying evaporation at the surface of a water body, energy transfer in a wet cooling tower, flow in a desert cooler, generating electric power, food processing, groves of fruit trees, crops damage because of freezing etc. There also molecular diffusion of species in the presence of chemical reaction within or at the boundary during several practical diffusive operations. There are two kinds of changes namely homogeneous and heterogeneous. A homogeneous reaction takes place uniformly in the entire given phase whereas a heterogeneous reaction exists in a restricted region or within the boundary of a phase. The smog formation is an important example representing a first order homogeneous chemical change. Many researchers in view of such facts are engaged for the discussion of flows with chemical reactions. . For-instance Seddeek and Almushigeh [1] studied the radiation and variable viscosity effects on MHD free convective over a stretching sheet in presence of chemical reaction. Kandasamy et al. [2] presented group analysis for Soret and Dufour effects on free convective heat and mass transfer with thermophoresis and chemical reaction over a porous stretching surface in the presence of heat source/sink. Pal and Talukdar [3] presented the combined effects of Joule heating and chemical reaction on unsteady magnetohydrodynamic mixed convection with viscous dissipation over a vertical plate in the presence of porous media and thermal radiation. Joneidi et al. [4] presented analytical treatment of MHD free convection flow over a stretching sheet with chemical reaction.Vyas [5] investigated the radiation effect on MHD flow over a nonisothermal stretching sheet in a porous medium.

The objective of this paper was to explore the effects of viscous dissipation, thermal radiation, heat generation and chemical reaction on MHD boundary layer flow over a non-isothermal stretching sheet embedded in porous medium. Using a similarity approach, the governing equations are transformed into nonlinear ordinary equations and solved numerically using shooting technique along with fourth-order RungeKutta method. The pertinent results are displayed graphically and discussed quantitatively.

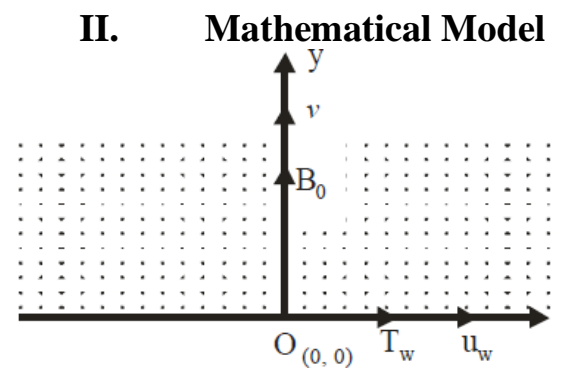

Figure A : Physical model 
Consider the steady two-dimensional forced convection boundary-layer flow of incompressible, viscous, electrically conducting fluid in a fluid saturated horizontal porous medium caused by linearly stretching sheet placed at the bottom of the porous medium. In the Cartesian co-ordinate system, the $x$-axis is along the sheet and $y$-axis is normal to the $x$-axis ( see Figure A). Two equal and opposite forces are applied along the sheet so that the wall is stretched, keeping the position of the origin fixed. The stretching velocity changes linearly with the distance from the origin. A uniform magnetic field of strength $B_{0}$ is applied normal to sheet. We assume that the wall temperature $T_{w}>T_{\infty}$, where $T_{\infty}$ is the uniform temperature of the ambient temperature. We supposed that the fluid is optically dense, Newtonian and without phase effect. Further it is assumed that the both the fluid and the porous medium are in local thermal equilibrium. We also consider that both the surroundings and the fluid are maintained at a constant temperature $T_{\infty}$ far away from the sheet. The Rosseland approximation is followed to describe the heat flux in the energy equation. Without considering the induced magnetic field, the external electric field, the electric field due to polarization of charges, ohmic and viscous dissipation, the governing boundary layer equations can be written as

$$
\begin{aligned}
& \frac{\partial u}{\partial x}+\frac{\partial u}{\partial y}=0 \\
& u \frac{\partial u}{\partial x}+v \frac{\partial u}{\partial y}=v \frac{\partial^{2} u}{\partial y^{2}}+g \beta\left(T-T_{\infty}\right)+g \beta^{*}\left(C-C_{\infty}\right)-\frac{\sigma B_{0}^{2}}{\rho} u-\frac{v}{k^{\prime}} u \\
& u \frac{\partial T}{\partial x}+v \frac{\partial T}{\partial y}=\frac{k}{\rho c_{p}} \frac{\partial^{2} T}{\partial y^{2}}-\frac{1}{\rho c_{p}} \frac{\partial q_{r}}{\partial y}+\frac{Q_{0}}{\rho c_{p}}\left(T-T_{\infty}\right)+\frac{\mu}{\rho c_{p}}\left(\frac{\partial u}{\partial y}\right)^{2} \\
& u \frac{\partial C}{\partial x}+v \frac{\partial C}{\partial y}=D \frac{\partial^{2} C}{\partial y^{2}}-K r^{*}\left(C-C_{\infty}\right)
\end{aligned}
$$

The boundary conditions for the velocity, temperature and concentration fields are

$u=c x, \quad v=0, \quad T=T_{w}=T_{\infty}+d x^{\alpha}, \quad C=C_{w}$ at $y=0$

$u \rightarrow 0, \quad T \rightarrow T_{\infty}, C \rightarrow C_{\infty}$ as $y \rightarrow \infty$

where $x$ and $y$ represents the coordinate axes along the continuous stretching surface in the direction of motion and normal to it, respectively; $u$ and $v$ are the velocity components along the $x$ and $y$ axes respectively, $v$ is the kinematics viscosity, $\mu$ is the fluid viscosity, $\sigma$ electric conductivity, $B_{0}$ is the uniform magnetic field, $\rho$ is the density, $k^{\prime}$ is the permeability of the porous medium, $c_{p}$ is the specific heat at constant pressure, $q_{r}$ is the radiation heat flux, $T$ is the temperature inside the boundary layer, $T_{\infty}$ is the temperature for away from the plate, $C$ is the species concentration in the boundary layer, $C_{\infty}$ is the concentration for away from the plate, $\beta$ is the coefficient of thermal expansion, $\beta^{*}$ is the coefficient of concentration expansion, $g$ is the gravitational acceleration, $\alpha$ is the wall temperature parameter, $Q_{0}$ is the volumetric rate of heat generation, $D$ is the molecular diffusivity of the species concentration, $c>0$ and $d$ are constants.

The radioactive heat flux $q_{r}$ is described by Roseland approximation for radiation such that

$$
q_{r}=-\frac{4 \sigma^{*}}{3 K^{*}} \frac{\partial T^{4}}{\partial y}
$$

Where $\sigma^{*}$ and $K^{*}$ are Stefan-Boltzmann constant and mean absorption coefficient, respectively. Following Chamkha [31], we assume that the temperature differences within the flow are sufficiently small so that $T^{4}$ can be expressed as a linear function after using Taylor series to expand $T^{4}$ about the free stream temperature $T_{\infty}$ and neglecting higher-order terms. This result is given by the approximation:

$T^{4} \approx 4 T_{\infty}^{3} T-3 T_{\infty}^{4}$

Using equation (6) and (7) in (3), we obtain

$$
\frac{\partial q_{r}}{\partial y}=-\frac{16 \sigma^{*}}{3 K} \frac{\partial^{2} T^{4}}{\partial y^{2}}
$$


We introduce the following non-dimensional variables:

$$
\begin{aligned}
& \eta=\sqrt{\frac{c}{v}} y, u=\frac{\partial \psi}{\partial y}=x c f^{\prime}, v=-\frac{\partial \psi}{\partial x}=-\sqrt{c v} f, M=\frac{\sigma B_{0}^{2}}{\rho c}, K=\frac{v}{k^{\prime} c}, \\
& \theta=\frac{T-T_{\infty}}{T_{w}-T_{\infty}}, \phi=\frac{C-C_{\infty}}{C_{w}-C_{\infty}}, R=\frac{K k^{*}}{4 \sigma T_{\infty}^{3}}, \operatorname{Pr}=\frac{\rho v c_{p}}{k}, \operatorname{Pr}=\frac{\rho v c_{p}}{k}, G r=\frac{g \beta\left(T_{w}-T_{\infty}\right)}{c^{2} x}, \\
& G c=\frac{g \beta^{*}\left(C_{w}-C_{\infty}\right)}{c^{2} x}, E c=\frac{c^{2} x^{2}}{c_{p}\left(T_{w}-T_{\infty}\right)}, Q=\frac{Q_{0}}{c \rho c_{p}}, S c=\frac{v}{D}, K r=\frac{K r^{*}}{c}
\end{aligned}
$$

In view of equation (6), the equations (2) - (4) take the form

$$
\begin{aligned}
& f^{\prime \prime \prime}+f f^{\prime \prime}-\left(f^{\prime}\right)^{2}+G r \theta+G c \phi-(M+K) f^{\prime}=0 \\
& \theta^{\prime \prime}-\left(\frac{3 \operatorname{Pr} R}{3 R+4}\right)\left[\alpha \theta f^{\prime}-f \theta^{\prime}\right]+\left(\frac{3 \operatorname{Pr} R}{3 R+4}\right) Q \theta+\left(\frac{3 \operatorname{Pr} R}{3 R+4}\right) E c\left(f^{\prime \prime}\right)^{2}=0 \\
& \phi^{\prime \prime}+S c f \phi^{\prime}-K r S c \phi=0
\end{aligned}
$$

where the primes denote the differentiation with respect to $\eta, M$ is the magnetic parameter, $K$ is the permeability parameter, $\operatorname{Pr}$ is the Prandtl number, $R$ is the radiation parameter, $\alpha$ is wall temperature parameter, $G r$ is the Grashof number, $G c$ is the modified Grashof number, $E c$ is the Eckert number, $Q$ is the heat generation parameter, $S c$ is the Schmidt number and $K r$ is the chemical reaction parameter.

The corresponding boundary conditions are

$$
\begin{array}{lll}
f^{\prime}=1, f=0, \theta=1, \phi=1 & \text { at } & \eta=0 \\
f^{\prime}=0, \theta=0, \phi=0 & \text { as } & \eta \rightarrow \infty
\end{array}
$$

\section{Numerical Computation}

The numerical solutions of the non-linear differential equations (9) - (11) under the boundary conditions (12) have been performed by applying a shooting method along with the fourth order Runge-Kutta method. First of all higher order non-linear differential equations (9) - (11) are converted into simultaneous linear differential equations of first order and they are further transformed into initial value problem by applying the shooting technique. From this process of numerical calculation, the Nusselt number ,skin-friction coefficient and Sherwood number which are respectively proportional to $f^{\prime \prime}(0),-\theta^{\prime}(0)$ and $-\phi^{\prime}(0)$ are also sorted out and their numerical values are presented in a tabular form.

\section{Results And Discussion}

To analyze the results, numerical computation has been carried out using the method described in the previous paragraph for various in governing parameters, namely, thermal Grashof number $G r$, modified Grashof number $G c$, the magnetic field parameter $M$, permeability parameter $K$, Prandlt number $P r$, wall temperature parameter $\alpha$, thermal radiation parameter $R$, heat generation parameter $Q$, Schmidt number $S c$, Eckert number $E c$, and chemical reaction parameter $K r$. In the present study following default parameter values are adopted for computations: $G r=2.0, G c=2.0, M=1.0, K=0.5, P r=0.72, R=1.0, \alpha=1.0, Q=0.5, E c=0.5, S c=0.6$, $K r=0$. All graphs therefore correspond to these values unless specifically indicated on the appropriate graph. The influence of the thermal Grashof number $G r$ on the velocity profile is presented in Figure 1. It is observed that there is a rise in the velocity due to enhancement of thermal buoyancy force. Figure 2 presents typical velocity profiles in the boundary layer for various values of the solutal Grashof number $G c$, while all remaining parameters are kept at some fixed values. As expected, the fluid velocity increases and the peak value is more distinctive due to increase in the species buoyancy force.

The effects of the magnetic field parameter $M$ on the dimensionless temperature, velocity and concentration fields are shown in Figs. 3- 5; it is obvious that an increase in the magnetic field parameter $M$ results in a decrease in the velocity. It is find that the temperature and concentration profiles increase with the increasing of magnetic field parameter $M$.

Figs. 6-8 show the dimensionless temperature, velocity, and concentration profiles for different values of permeability parameter $K$. it can be find that the velocity profiles decrease with the increase of permeability parameter $K$. it is observed that the temperature and concentration profiles increase of permeability parameter $K$. 


\section{Graphs And Tables}

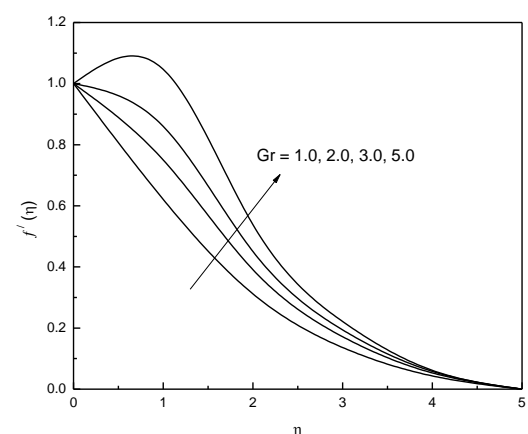

Fig.1. Velocity profiles for different values of $\boldsymbol{G r}$

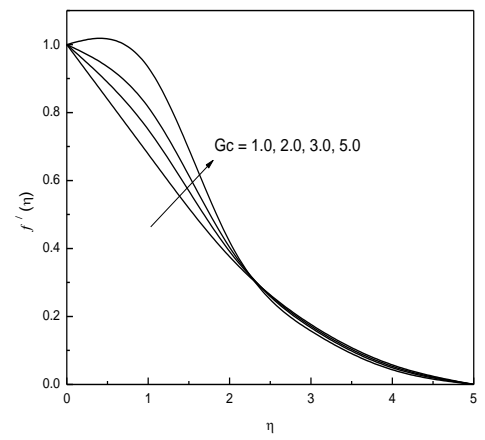

Fig.2. Velocity profiles for different values of $G c$

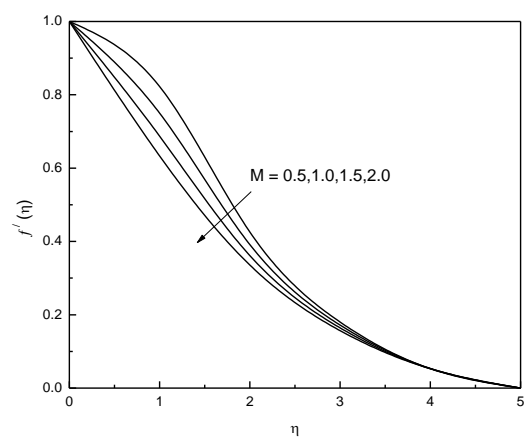

Fig.3. Velocity profiles for different values of $M$

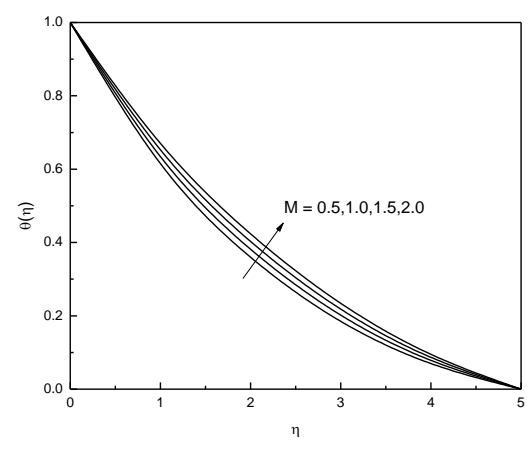

Fig.4. Temperature profiles for different values of $M$

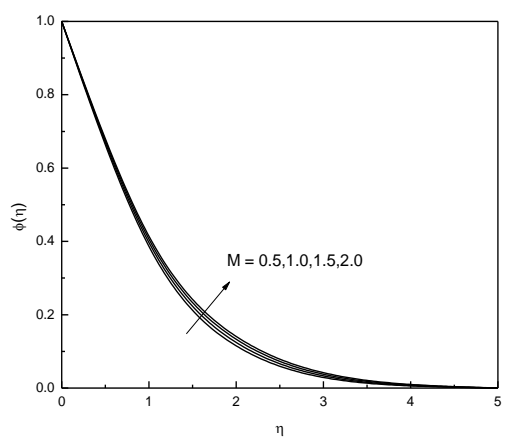

Fig.5.Concentration profiles for different values of $\boldsymbol{M}$

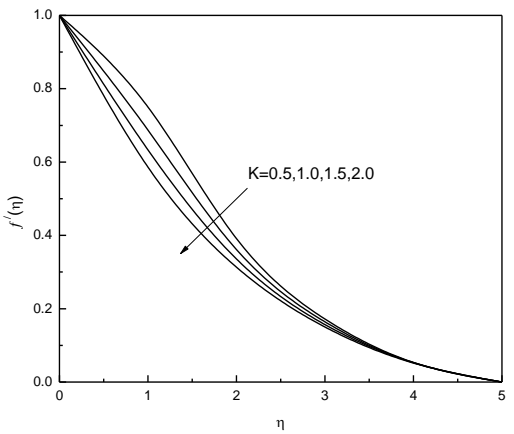

Fig.6. Velocity profiles for different values of $K$ 


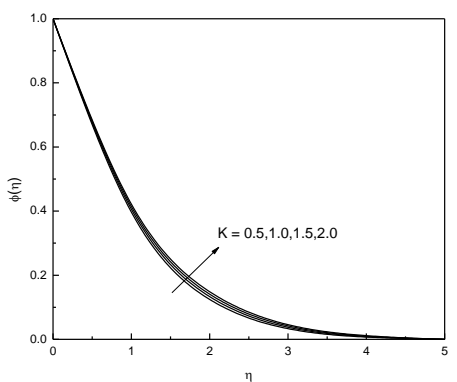

Fig.7. Temperature profiles for different values of $K$

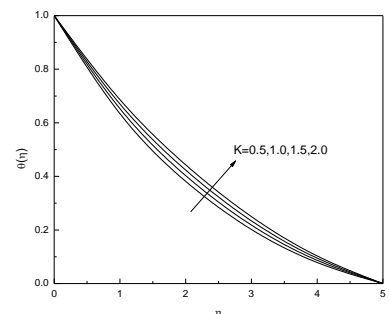

Fig.8.Concentration profiles for different values of $K$

Table 1 :

\begin{tabular}{|c|c|c|c|c|c|c|}
\hline$G r$ & $G c$ & $M$ & $K$ & $f^{\prime \prime}(0)$ & $-\theta^{\prime}(0)$ & $-\phi^{\prime}(0)$ \\
\hline 2.0 & 2.0 & 1.0 & 0.5 & 0.150762 & 0.442613 & 0.777892 \\
\hline 3.0 & 2.0 & 1.0 & 0.5 & 0.553446 & 0.482143 & 0.798155 \\
\hline 4.0 & 2.0 & 1.0 & 0.5 & 0.990722 & 0.505879 & 0.816113 \\
\hline 2.0 & 3.0 & 1.0 & 0.5 & 0.497064 & 0.463111 & 0.79163 \\
\hline 2.0 & 4.0 & 1.0 & 0.5 & 0.835841 & 0.475835 & 0.804472 \\
\hline 2.0 & 2.0 & 2.0 & 0.5 & -0.270377 & 0.388834 & 0.755957 \\
\hline 2.0 & 2.0 & 3.0 & 0.5 & -0.629993 & 0.336638 & 0.738372 \\
\hline 2.0 & 2.0 & 1.0 & 1.0 & -0.068862 & 0.415729 & 0.766295 \\
\hline 2.0 & 2.0 & 1.0 & 2.0 & -0.456655 & 0.362382 & 0.746699 \\
\hline
\end{tabular}

Table 2:

\begin{tabular}{|c|c|c|c|c|c|c|c|}
\hline$P r$ & $R$ & $\alpha$ & $E c$ & $Q$ & $f^{\prime \prime}(0)$ & $-\theta^{\prime}(0)$ & $-\phi^{\prime}(0)$ \\
\hline 0.72 & 1.0 & 1.0 & 0.5 & 0.5 & 0.150762 & 0.442613 & 0.777892 \\
\hline 1.0 & 1.0 & 1.0 & 0.5 & 0.5 & 0.119472 & 0.517432 & 0.77389 \\
\hline 1.5 & 1.0 & 1.0 & 0.5 & 0.5 & 0.0729682 & 0.635259 & 0.768052 \\
\hline 0.72 & 1.5 & 1.0 & 0.5 & 0.5 & 0.131292 & 0.488779 & 0.775396 \\
\hline 0.72 & 2.0 & 1.0 & 0.5 & 0.5 & 0.118641 & 0.519463 & 0.773784 \\
\hline 0.72 & 1.0 & 1.5 & 0.5 & 0.5 & 0.11505 & 0.55654 & 0.774103 \\
\hline 0.72 & 1.0 & 2.0 & 0.5 & 0.5 & 0.0850994 & 0.656825 & 0.770945 \\
\hline 0.72 & 1.0 & 1.0 & 0.7 & 0.5 & 0.178787 & 0.373412 & 0.781302 \\
\hline 0.72 & 1.0 & 1.0 & 1.0 & 0.5 & 0.229465 & 0.252805 & 0.787442 \\
\hline 0.72 & 1.0 & 1.0 & 0.5 & 0.7 & 0.155644 & 0.433096 & 0.778502 \\
\hline 0.72 & 1.0 & 1.0 & 0.5 & 1.0 & 0.162861 & 0.419117 & 0.779407 \\
\hline
\end{tabular}

Table 3:

\begin{tabular}{|l|l|l|l|l|}
\hline $\mathrm{Sc}$ & $\mathrm{Kr}$ & $f^{\prime \prime}(0)$ & $-\theta^{\prime}(0)$ & $-\phi^{\prime}(0)$ \\
\hline 0.6 & 0.5 & 0.150762 & 0.442613 & 0.777892 \\
\hline 0.78 & 0.5 & 0.116005 & 0.433268 & 0.89045 \\
\hline 1.0 & 0.5 & 0.0816344 & 0.425098 & 1.01176 \\
\hline 0.6 & 1.0 & 0.110634 & 0.433838 & 0.94824 \\
\hline 0.6 & 1.5 & 0.0794772 & 0.427467 & 1.09362 \\
\hline
\end{tabular}

\section{References}

[1] M.A. Seddeek , and A.A. Almushigeh A.A, Effects of radiation and variable viscosity on MHD free convective flow and mass transfer over a stretching sheet with chemical reaction, Appl. Appl Math, Vol. 5, No.1,2010, pp. 181-197.

[2] R.Kandasamy, T.Hayat, S.Obaidat S, Group theory transformation for Soret and Dufour effects on free convective heat and mass transfer with thermophoresis and chemical reaction over a porous stretching surface in the presence of heat source/sink, Nucl. Eng. Des., Vol. 241,No.6,2011, pp. 2155-2161.

[3] D.Pal, and B.Talukdar, Combined effects of Joule heating and chemical reaction on unsteady magnetohydrodynamic mixed convection of a viscous dissipating fluid over a vertical plate in porous media with thermal radiation, Math. Comput. Modelling, Vol. 54, No.11,2011,pp. 3016-3036.

[4] A.A. Joneidi, G.Domairry, and M. Babaelahi, Analytical treatment of MHD free convective flow and mass transfer over a stretching sheet with chemical reaction, J. Taiwan Inst. Chem. Eng., Vol. 41No.1,,2010, pp. 35-43, 2010.

[5] P. Vyas, Radiative MHD flow over a non-isothermal stretching sheet in a porous medium, Applied Mathematical Sciences, Vol. 4, No. 50,2010, pp. 2475-2484. 